\title{
Cooperation of Multiple Robots to Solve Maze Tasks
}

\author{
Claudio R. F. Costa ${ }^{1}$ and Eliana P. L. Aude ${ }^{2}$ \\ ${ }^{1}$ IME - Military Institute of Engineering, Rio de Janeiro, Brazil \\ ${ }^{2}$ NCE/Federal University of Rio de Janeiro, PO Box 2324, RJ, 20001-970, Brazil \\ elaude@nce.ufrj.br
}

\begin{abstract}
This paper discusses the use of cooperation and distributed intelligence within a robot team to solve a maze problem in which a set of ordered control point tasks has to be performed before the robots exit the maze. At first, the maze structure and the control point locations are unknown. While the maze is explored, each robot dynamically maps the maze structure with information provided by its sensors and sent by the other robots. Within each robot, a dynamic scheduler uses the gathered information to update the robot goal.
\end{abstract}

Keywords: distributed intelligence, maze problem, dynamic scheduling

Research on robot teams has led to great progress in the quality of the solution for many problems $[1,2,3]$. In this work, a maze exploration by a robot team is considered. The maze contains a set of ordered control points, each one with an associated task. At first, the maze structure and the control point locations are unknown. The robots cannot exit the maze before all control point tasks have been performed in the correct order. They cooperate to map the maze structure and to locate the control points by exchanging information. At each maze position, the information sent by a robot consists of the position coordinates and status in each direction (free or blocked). The message also contains information on the exit location and on the control point locations and status.

A simulator has been developed to study the problem. The maze structure is gradually displayed on the screen as the maze exploration by the robots evolves. Fig.1 shows a simulator screen snapshot. The robots are the small numbered squares, indicating the order they entered the maze. The control points are represented by larger numbered squares, which indicate their task execution order.

Each robot performs a depth-first maze search while the robot team as a whole performs a breadth-first search. In this process, each robot dynamically maps the maze structure and is able to find which is the closest robot to a target control point. When a robot reaches a junction and chooses a direction that leads it to a dead end, the status of all the maze cells along that way is set to blocked in the direction towards the blockage and to free in the opposite direction. So, if another robot reaches the same junction, it won't take the path leading to that dead end and any robot that had already taken that path moves back to the junction.

When a robot finds a control point which is not the one in turn to have its task performed, three behavior models can be adopted. In the first one, the robot continues the maze search and when a located control point is due to have its task performed, the closest robot goes to it along the shortest known path. The second 
behavior is similar except that a robot will stay still at a control point if it is the one associated with the next task to be performed. In the third behavior, a robot always stays at a located control point position waiting for the right time to perform its task. With the first two behaviors, when a located control point is due to have its task performed, the closest robot may have to interrupt the maze search and go to it. For that, each robot has its own dynamic task scheduler, which permanently updates the robot goal and avoids conflicts between different robot goals.

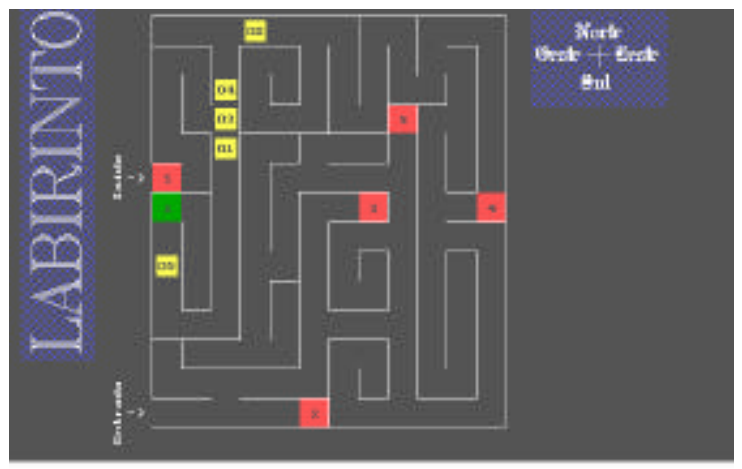

CONT ROLAB-NCE( UFRJ)

Fig. 1. Simulator screen snapshot

Simulation experiments have been performed with the use of different maze sizes, robot team sizes, maze search models and numbers of control points. Wireless robot communication bandwidth was always set to $2 \mathrm{Mbps}$ and the robots were assumed to move at $0.5 \mathrm{~m} / \mathrm{s}$ as they followed the Controlab AGV [4] model. Simulation results show that the third behavior model is superior when the number of robots is much larger than the number of control points. For any maze size, the time spent on solving the problem is reduced at a faster rate when the number of robots increases within a small sized team. When the maze problem requires all the robots to exit the maze instead of a single one, the solution becomes slower for large robot teams due to long exit queues and maze path saturation.

\section{Acknowledgements}

The authors acknowledge the support given by CNPq/RHAE, Brazil, and the Brazilian Navy to the development of this research work.

\section{References}

1. R. Alami, S. Fleury, M. Herrb, F. Ingrand, F. Robert. (1998) Multi Robot Cooperation in the Martha Project. IEEE Robotics and Automation Magazine, 5, No. 1, 1-21.

2. B. Yamauchi. (1998) Frontier-Based Exploration Using Multiple Robots, Proceedings of the Autonomous Agents 98.

3. L. Parker. (1994) Heterogeneous Multi-Robot Cooperation, Ph.D. Thesis, MIT.

4. E. Aude, G. Carneiro, H. Serdeira, J. Silveira, M. Martins, E. Lopes. (1999) Controlab MUFA: A Multi-level Fusion Architecture for Intelligent Navigation of a Tele-robot, Proceedings of the IEEE Int'l Conference on Robotics and Automation, 465-472. 